\title{
CHÂT LƯợNG CUỘC SỐNG VÀ CÁC YẾU TỐ LIÊN QUAN TRÊN BỆNH NHÂN SUY TĨNH MẠCH MẠN TÍNH CHI DƯỚI
}

Nguyễn Thị Thanh Thuần*, Nguyễn Hoài Nam**

\section{TÓM TẮT}

Đặt vấn đề: Suy tĩnh mạch mạn tính chi dưới (STMMTCD) là vấn đề toàn cầu. Bệnh ảnh hưởng lớn lên chất lượng cuộc sống (CLCS), là gánh nặng cho quốc gia - y tế. Thực trạng tại Việt Nam CLCS bệnh nhân chưa được quan tâm. Nghiên cứu khảo sát CLCS và các yếu tố liên quan trên bệnh nhân $\mathrm{STMMTCD}$ nhằm nâng cao chất lượng chăm sóc cũng như sức khỏe bệnh nhân.

Mục tiêu: Xác định điểm số CLCS và các yếu tố liên quan trên bệnh nhân STMMTCD.

Phương pháp nghiên cứu: tiền cứu mô tả cắt ngang, khảo sát trên 68 người STMMTCD được chọn ngẫu nhiên, tuổi từ 18 đến khám tại BV Đại học Y Dược. Phỏng vấn trực tiếp bệnh nhân, sử dụng bộ câu hỏi SF-36. Phân tích hồi quy tuyến tính đa biến đo lường mối liên quan giữa CLCS và các yếu tố.

Kết quả: Bệnh nhân STMMTCD có điểm số sức khỏe thể chất $(51,56 \pm 14,53)$, sức khỏe tâm thần $(53,91 \pm 15,20)$, CLCS chung $(52,74 \pm 10,30)$. Yếu tố liên quan đến CLCS gồm giới, thu nhập, phân loại lâm sàng CEAP, vận động thể lực vừa sức và mang vớ $\mathrm{y}$ khoa.

Kết luận: CLCS bệnh nhân STMMTCD bị suy giảm, cả thể chất lẫn tâm thần. Cần khuyến khích bệnh nhân vận động thể lực vừa sức và mang vớ y khoa giúp cải thiện CLCS.

Từ khóa: Suy tĩnh mạch mạn tính chi duới, Chất lự̛ng cuộc sống, SF-36.

\section{SUMMARY}

QUALITY OF LIFE AND ITS ASSOCIATED FACTORS

\section{AMONG PATIENTS WITH CHRONIC VENOUS INSUFFICIENCY}

Background: Chronic venous insufficiency (CVI) is a global problem. Disease affects the quality of life (QOL); is a burden for the Nation. In Vietnam, QOL among the CVI patients has not yet been considered. This study on QOL and its associated factors among CVI patients to improve quality of care as well as health of patients.

Objectives: To determine score of QOL and its associated factors among CVI patients

Methods: A retrospective descriptive cross-sectional study, 68 CVI patients from 18 years old were randomly selected to see the University of medicine and pharmacy HCM City. The data were collected during face-to-face interviews using SF-36 scale. Multivariable linear regression analyses were used to measure the association between QOL and variables.

Results: The mean scores in physical health was $(51,56 \pm 14,53)$, mental health was $(53,91 \pm 15,20)$, QOL was $(52,74 \pm 10,30)$. Factors associated with QOL among CVI patients were gender, income level, clinical classification CEAP, physical activity, compression stocking.

\footnotetext{
* Bộ môn Điều duõng - ĐH Công Nghệ Đồng Nai **Bộ môn Ngoại lồng ngưc tim mach - ĐH Y Dượ TP. HCM Ngưòi chịu trách nhiệm khoa học: PGS.TS Nguyễn Hoài Nam Ngày nhận bài: 01/02/2019 - Ngày Cho Phép Đăng: 23/03/2020 Phản Biện Khoa học: PGS.TS. Đặng Ngocc Hùng GS.TS. Lê Ngoc Thành
} 
Conclusion: QOL in CVI patients were decreased, both physically and mentally. Encouraging moderate physical activity and compression stocking to improve QOL for CVI patients.

Keywords: Chronic venous insufficiency, quality of life, SF-36.

\section{I. ĐẶT VẤN ĐỀ}

STMMTCD là bệnh mạn tính, gây ra hàng loạt các triệu chứng khó chịu, đặc biệt khả năng tàn phế giai đoạn cuối bệnh và tử vong do thuyên tắc động mạch phổi ${ }^{[1]}$. Bệnh là vấn đề toàn cầu, với tỷ lệ ngày càng gia tăng đỉnh điểm lên tới $71 \%{ }^{[5]}$, đặc biệt ở các nước Phương Tây với nền công nghiệp hóa, tại Mỹ tỷ lệ này là 40\% ${ }^{[7]}$.

Vấn đề phức tạp bởi thực tế STMMTCD là bệnh tiến triển và tái diễn, đòi hỏi quá trình điều trị khó khăn, tốn kém, cần kiên trì của cả bệnh nhân và thầy thuốc. Bệnh làm giảm thẩm mỹ, giảm khả năng lao động, tăng số người nghỉ hưu sớm do đó làm giảm CLCS và là gánh năng tài chính cho quốc gia - xã hội. Các nghiên cứu trên thế giới đã tìm thấy STMMTCD làm suy giảm CLCS, ảnh hưởng đến sức khỏe thể chất lẫn tâm thần ${ }^{[4],[11],[13],[19] . ~}$

Tại Việt Nam, cùng với sự phát triển kinh tế và thay đổi lối sống của người dân thì tỷ lệ bệnh ngày càng gia tăng và có xu hướng trẻ hóa. Hiện nay các nghiên cứu về CLCS ở nhóm bệnh này vẫn chưa được quan tâm, do đó nghiên cứu được tiến hành với mong muốn góp một phần vào việc đánh giá toàn diện bệnh, từ đó là cơ sở cho chăm sóc điều dưỡng nhằm nâng cao sức khỏe cũng như chất lượng cuộc sống bệnh nhân.

\section{Mục tiêu nghiên cứu}

Xác định điểm số chất lượng cuộc sống bệnh nhân suy tĩnh mạch mạn tính chi dưới trong nhóm nghiên cứu theo thang đo SF-36.
Xác định một số yếu tố liên quan đến chất lượng cuộc sống trên bệnh nhân suy tĩnh mạch mạn tính chi dưới

\section{II. ĐỐI TƯợNG VÀ PHƯONG PHÁP NGHIÊN CÚ'U}

\section{1. Đối tượng nghiên cứu}

Chọn mẫu ngẫu nhiên hệ thống từ những bệnh nhân nghi ngờ STMMTCD đến khám tại Phòng khám Ngoại Lồng ngực - Mạch máu BV Đại học Y dược từ 3/2018 đến 6/2018

\subsubsection{Tiêu chuẩn lựa chọn bệnh nhân}

Nam hoặc nữ $\geq 18$ tuổi, chẩn đoán STMMTCD, tự nguyện tham gia vào nghiên cứu.

2.1.2. Tiêu chuẩn loại trừ: Bệnh nhân tái khám từ lần thứ 2 trở đi trong đợt thu thập số liệu, đang mắc các bệnh cấp tính, bệnh nặng, phụ nữ đang mang thai, đang mắc từ hai bệnh mạn tính trở lên, không hợp tác được.

\subsection{Phương pháp Nghiên cứu}

\subsubsection{Thiết kế nghiên cứu}

Tiền cứu, mô tả cắt ngang

\subsubsection{Cõ̃ mẫu}

Dựa vào công thức ước lượng một trung bình với khoảng tin cậy $95 \%$, sai số $2 \%$, độ lệch chuẩn 8,37 theo nghiên cứu Soydan $(2016)^{[19]}$. Cơ mẫu cần thiết là 68 bệnh nhân.

\subsubsection{Phương pháp thu thập số liệu}

Phỏng vấn trực tiếp bệnh nhân sử dụng bộ câu hỏi SF-36.

SF-36 gồm 36 câu hỏi tập trung 2 lĩnh vực sức khỏe thể chất (SKTC) và sức khỏe tâm thần (SKTT). Đánh giá CLCS theo thang điểm từ 0 đến 100 với CLCS tăng dần, chia làm 4 mức độ: 0-25 điểm: CLCS kém, 26-50 điểm: CLCS trung bình kém, 51- 75 điểm: CLCS trung bình khá, 76100 điểm: CLCS khá, tốt ${ }^{[2]}$. 


\subsubsection{Phương pháp phân tích số liệu:}

Các biến phụ thuộc là điểm trung bình SKTC, SKTT, CLCS theo thang đo SF-36. Các biến độc lập bao gồm đặc điểm nhóm nghiên cứu gồm tuổi, giới tính, mức thu nhập, mang vớ $\mathrm{y}$ khoa, vận động thể lực, phân loại lâm sàng CEAP,,.

Xử lý số liệu bằng phần mềm SPSS20.0. Phép kiểm Kolmogorov - Smirnov xác định điểm SKTC, SKTT, CLCS có phân phối chuẩn và gần chuẩn. Thống kê mô tả bao gồm trung bình \pm độ lệch chuẩn và tần số (tỷ lệ \%). Thống kê phân tích để xác định mối liên quan giữa điểm SKTC, SKTT, CLCS với các biến số nhóm nghiên cứu. Trong đó, phân tích đơn biến gồm kiểm định t-test độc lập và ANOVA. Sau đó, các biến số khi xét mối liên quan trong phân tích đơn biến có $\mathrm{p} \leq 0,2$ được đưa vào phân tích hồi quy tuyến tính đa biến nhằm xác định các yếu tố thực sự liên quan đến điểm SKTC, SKTT và CLCS. Sự khác biệt có ý nghĩa thống kê với $\mathrm{p}<0,05$.

\section{KẾT QUẢ NGHIÊN CÚU}

Qua nghiên cứu trên 68 bệnh nhân STMMTCD tại BV Đại học Y dược. Các kết quả ghi nhận được như sau:

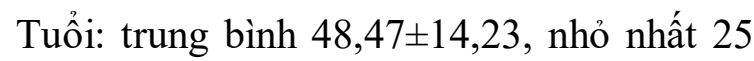
tuổi, lớn nhất 78 tuổi.

Giới: 72,1\% là nữ, tỷ lệ nữ/nam khoảng 2,6/1.

\section{1. Điểm trung bình CLCS bệnh nhân theo thang đo SF-36}

Bảng 1. Điểm trung bình CLCS bệnh nhân ( $n=68)$

\begin{tabular}{|l|c|c|c|}
\hline Lĩnh vục & TB \pm ĐLC & GTLN & GTNN \\
\hline SKTC & $51,56 \pm 14,53$ & 83 & 21 \\
\hline SKTT & $53,91 \pm 15,20$ & 89 & 18 \\
\hline CLCS & $52,74 \pm 10,30$ & 82 & 29 \\
\hline
\end{tabular}

Bệnh nhân STMMTCD có điểm trung bình SKTC thấp hơn SKTT. Điểm SKTC, SKTT, CLCS đều ở mức trung bình khá.

\subsection{Các yếu tố dự báo liên quan đến CLCS bệnh nhân STMMTCD}

Bảng 2. Các yếu tố liên quan đến CLCS bệnh nhân STMMTCD sau phân tích đa biến (n=68)

\begin{tabular}{|c|c|c|c|c|c|c|}
\hline \multirow[t]{3}{*}{ Nội dung } & \multicolumn{6}{|c|}{ Điểm CLCS } \\
\hline & \multicolumn{2}{|c|}{ SKTC } & \multicolumn{2}{|c|}{ SKTT } & \multicolumn{2}{|c|}{ CLCS } \\
\hline & Hệ số & KTC 95\% & Hệ số & KTC 95\% & Hệ số & KTC 95\% \\
\hline $\begin{array}{l}\text { Giới } \\
\text { Nam } \\
\text { Nữ }\end{array}$ & & & $-7,47^{*}$ & $-14.57-(-0,37)$ & $-7,16^{* * *}$ & $-11,05-(-3,26)$ \\
\hline $\begin{array}{l}\text { Mức thu nhập } \\
\text { Hộ không nghèo } \\
\text { Hộ nghèo }\end{array}$ & & & $-17,95^{*}$ & $-32,45-(-3,46)$ & & \\
\hline $\begin{array}{l}\text { Mang vớ y khoa } \\
\text { Không } \\
\text { Có }\end{array}$ & $17,91 * * *$ & $11,93-23,90$ & & & $13,29 * * *$ & $9,67-16,91$ \\
\hline
\end{tabular}




\begin{tabular}{|l|l|l|l|l|l|l|}
\hline $\begin{array}{l}\text { Vận động thể lụ̣c } \\
\text { Không } \\
\text { Có }\end{array}$ & & & $10,19^{* *}$ & $3,72-16,67$ & $4,63 *$ & $0,93-8,33$ \\
\hline $\begin{array}{l}\text { Phân loại lâm } \\
\text { sàng CEAP } \\
\text { C1-C2 } \\
\text { C3-C4-C6 }\end{array}$ & & & $-10,40 * *$ & $-17,08-(-3,72)$ & $-5,74 * *$ & $-9,57-(-1,92)$ \\
\hline
\end{tabular}

${ }^{*} p \leq 0,05, * * P \leq 0,01, * * * p \leq 0,0001$ (Hồi quy tuyến tính đa biến)

Sau phân tích hồi quy tuyến tính đa biến về một số đặc điểm nhóm nghiên cứu, tìm thấy giới nữ có tác động tiêu cực, có điểm số SKTT $(\mathrm{p}<0,05)$, CLCS chung $(\mathrm{p}<0,001)$ thấp hơn giới nam. Bệnh nhân thuộc độ lâm sàng CEAP nặng (C3-C4-C6) tác động tiêu cực, có điểm số SKTT $(\mathrm{p}<0,01)$, CLCS chung $(\mathrm{p}<0,01)$ thấp hơn nhóm độ nhẹ $(\mathrm{C} 1-\mathrm{C} 2)$. Một tác động tiêu cực nữa là bệnh nhân có thu nhập thấp (hộ nghèo/ cận nghèo) có điểm số SKTT thấp $(\mathrm{p}<0,05)$ hơn nhóm không nghèo. Ở một khía cạnh ngược lại, tác động tích cực từ mang vớ y khoa lên điểm số SKTC $(p<0,0001)$, CLCS chung $(p<0,001)$ và vận động thể lực vừa sức lên điểm số $\mathrm{SKTT}$ $(\mathrm{p}<0,01)$, CLCS chung $(\mathrm{p}<0,05)$ cao hơn nhóm còn lại.

\section{BÀN LUẬA}

Nghiên cứu này đánh giá CLCS bệnh nhân STMMTCD, kết quả tìm thấy bệnh nhân có điểm SKTC, SKTT thấp và CLCS bị suy giảm. Điểm trung bình SKTC $(51,56 \pm 14,53)$, SKTT $(53,91 \pm 15,20)$ và $\operatorname{CLCS}(52,74 \pm 10,30)$ đều nằm ở mức trung bình khá. Tương tự nhiều nghiên cứu đã báo cáo rằng $\mathrm{STMMTCD}$ làm suy giảm đáng kể SKTC, SKTT, trong đó SKTC bị suy giảm trầm trọng hơn $\mathrm{SKTT}^{[9],[11],[13],[19]}$. Bệnh STMMTCD làm giới hạn chức năng, gây hạn chế trong các hoạt động hằng ngày, tác động tới tâm lý bởi triệu chứng khó chịu, các biến đổi về thẩm mỹ, biến chứng do đó bệnh ảnh hưởng tới tất cả các khía cạnh của cuộc sống, từ đó CLCS bệnh nhân bị suy giảm.
Trong nghiên cứu tìm thấy giới có liên quan đến CLCS bệnh nhân STMMTCD. Các nghiên cứu khác cho kết quả tương tự giới nữ có CLCS thấp hơn nam ${ }^{[11],[13]}$. Ngược lại ở nghiên cứu Soydan $^{[19]}$ chưa tìm thấy sự khác biệt CLCS ở hai giới. Sự khác biệt CLCS ở giới có thể do nam có sức chịu đựng cao, nhận thức về tình trạng sức khỏe, cuộc sống lạc quan, tích cực hơn trong khi nữ thường lo lắng, suy nghĩ nhiều và bi quan về bệnh tật.

Theo Ruckley và Valencia ${ }^{[18],[20]}$ STMMTCD có tác động tiêu cực đến kinh tế bệnh nhân và ngược lại, liên quan đến việc chăm sóc dài hạn các tổn thương mãn tính, đặc biệt là loét. Nghiên cứu này, tìm thấy ở nhóm thu nhập thấp (hộ nghèo/cận nghèo) có điểm SKTT thấp hơn nhóm thu nhập trung bình và cao. Có thể thấy STMMTCD làm suy giảm khả năng lao động, đòi hỏi chi phí cao quá trình khám - điều trị lâu dài, liên tục nên đối với những hộ thu nhập thấp đây thật sự là một vấn đề lớn.

Nhiều nghiên cứu trên thế giới đã chỉ ra rằng phân loại lâm sàng $\mathrm{CEAP}$ càng nặng thì CLCS bệnh nhân STMMTCD càng giảm $^{[9],[11],[13],[19]}$. Tương tự, nghiên cứu này tìm thấy ở độ lâm sàng CEAP nặng (C3-C4-C6) có điểm SKTT và CLCS thấp hơn độ nhẹ (C1-C2). Nghiên cứu chưa tìm thấy mối liên quan với SKTC, có thể do gần một nữa đối tượng chúng tôi ở độ nhẹ $\mathrm{C} 1$, mà theo Rossi ${ }^{[17]}$ ở giai đoạn nhẹ chủ yếu ảnh hưởng tới SKTT. Như vậy, STMMTCD ở giai đoạn càng muộn thì SKTC 
càng giảm, ảnh hưởng đến công việc, sinh hoạt, các mối quan hệ gia đình - xã hội; về SKTT ở giai đoạn đầu bệnh nhân mệt mỏi, khó chịu, giai đoạn tiến triển xuất hiện các thay đổi ở da, loét làm bệnh nhân mất tự tin vào bản thân, ảnh hưởng nhiều đến cảm xúc - tâm lý.

Liệu pháp mang vớ y khoa là phương pháp điều trị được khuyến cáo nhiều nhất cho STMMTCD trong các hướng dẫn quốc tế[14]. Trên thế giới, các nghiên cứu đã tìm thấy hiệu quả của vớ $\mathrm{y}$ khoa trong cải thiện CLCS bệnh nhân ${ }^{[3],[16],[21]}$. Nghiên cứu này cho kết quả tương tự, nhóm tuân thủ mang vớ y khoa có điểm SKTC và $\mathrm{CLCS}$ cao hơn hẵn nhóm không mang vớ. Trong STMMTCD vớ y khoa giúp hỗ trợ chân, làm giảm đường kính tĩnh mạch, khép kín các van, ngăn chặn máu chảy xuống phần thấp của chân, từ đó giảm các triệu chứng và ngăn tiến triển bệnh.

Suy giảm chức năng bơm của cơ bắp chân có liên quan đến tiến triển của bệnh STMMTCD. Nhiều nghiên cứu đã cho kết quả sức khỏe được cải thiện thông qua các bài tập có giám sát $^{[10],[12],[15],[19]}$. Và mặc dù bệnh nhân STMMTCD đa số là người lớn tuổi, nhưng liệu pháp vận động vẫn có hiệu quả ${ }^{[8]}$. Campbel1 ${ }^{[6]}$ khuyến cáo đi bộ là một trong những biện pháp vận động ngăn quá trình tiến triển giãn tĩnh mạch. Trong nghiên cứu, bệnh nhân vận động thể lực vừa sức có điểm SKTT, CLCS cao hơn hẵn nhóm không vận động hoặc vận động mức quá nhẹ, quá nặng. Có thể thấy vận động thể lực vừa phải có khả năng tăng sức mạnh cơ bắp chân, tăng huyết động tĩnh mạch ở chi dưới, cải thiện khả năng vận động, giảm các triệu chứng bệnh; thêm nữa giúp tinh thần thoải mái, giảm stress, từ đó tăng CLCS bệnh nhân.

\section{KẾT LUẬN - KIẾN NGH!}

CLCS bệnh nhân STMMTCD bị suy giảm ở tất cả các thành phần thuộc sức khỏe thể chất và tâm thần. Do đó trong công tác chăm sóc, điều dưỡng khi tiếp cận bệnh nhân STMMTCD cần quan tâm chăm sóc cả về tinh thần chứ không hẵn chỉ cần chăm sóc thể chất.

Các yếu tố giới nữ, thu nhập thấp, độ lâm sàng nặng có tác động tiêu cực đến CLCS bệnh nhân STMMTCD, do đó cần chú ý hơn những đối tượng này trong chăm sóc. Ngược lại, mang vớ $\mathrm{y}$ khoa và vận động thể lực vừa sức có tác động tích cực làm tăng CLCS bệnh nhân, do vậy người điều dưỡng trong quá trình tư vấn, giáo dục sức khỏe cần nhấn mạnh và khuyến khích bệnh nhân tuân thủ điều trị mang vớ y khoa cũng như thay đổi lối sống bao gồm vận động thể lực, nhằm đạt mục đích cải thiện CLCS.

\section{TÀI LIỆU THAM KHẢO}

1. Phạm Khuê, Phạm Thắng (1998), "Chẩn đoán suy tĩnh mạch chi dưới", NXB Y học, Hà Nội, pp. 47-107.

2. Ware J. E., Jr. (2000), "SF-36 health survey update", Spine (Phila Pa 1976), 25 (24), pp. 3130-9.

\section{Andreozzi G. M., Cordova R.,} Scomparin M. A., et al. (2005), "Effects of elastic stocking on quality of life of patients with chronic venous insufficiency. An Italian pilot study on Triveneto Region", Int Angiol, 24 (4), pp. 325-9.

4. Andreozzi G. M., Cordova R. M., Scomparin A., et al. (2005), "Quality of life in chronic venous insufficiency. An Italian pilot study of the Triveneto Region", Int Angiol, 24 (3), pp. 272-7.

5. Beebe-Dimmer J. L., Pfeifer J. R., Engle J. S., et al. (2005), "The epidemiology of chronic venous insufficiency and varicose veins", Ann Epidemiol, 15 (3), pp. 175-84. 
6. Campbell W. B., Decaluwe H., Boecxstaens V., et al. (2007), "The Symptoms of Varicose Veins: Difficult to Determine and Difficult to Study", European Journal of Vascular and Endovascular Surgery, 34 (6), pp. 741-744.

7. Clark A., Harvey I., Fowkes F. G. (2010), "Epidemiology and risk factors for varicose veins among older people: crosssectional population study in the UK", Phlebology, 25 (5), pp. 236-40.

8. Fiatarone M. A., Marks E. C., Ryan N. D., et al. (1990), "High-intensity strength training in nonagenarians. Effects on skeletal muscle", Jama, 263 (22), pp. 3029-34.

9. Kahn S. R., M'Lan C E., Lamping D. L., et al. (2004), "Relationship between clinical classification of chronic venous disease and patient-reported quality of life: results from an international cohort study", J Vasc Surg, 39 (4), pp. 823-8.

10. Kan Y. M., Delis K. T. (2001), "Hemodynamic effects of supervised calf muscle exercise in patients with venous leg ulceration: a prospective controlled study", Arch Surg, 136 (12), pp. 1364-9.

11. Kaplan R. M., Criqui M. H., Denenberg J. O., et al. (2003), "Quality of life in patients with chronic venous disease: San Diego population study", J Vasc Surg, 37 (5), pp. 1047-53.

12. Klyscz T., Junger M., Junger I., et al. (1997), "Vascular sports in ambulatory therapy of venous circulatory disorders of the legs. Diagnostic, therapeutic and prognostic aspects", Hautarzt, 48 (6), pp. 384-90.

13. Kurz X., Lamping D. L., Kahn S. R., et al. (2001), "Do varicose veins affect quality of life? Results of an international population-based study", J Vasc Surg, 34 (4), pp. 641-8.

14. Nicolaides A. N. (2000), "Investigation of chronic venous insufficiency: A consensus statement (France, March 5-9, 1997)", Circulation, 102 (20), pp. E126-63.

15. Padberg Jr F. T., Johnston M. V., Sisto S. A. (2004), "Structured exercise improves calf muscle pump function in chronic venous insufficiency: a randomized trial", Journal of Vascular Surgery, 39 (1), pp. 79-87.

16. Prandoni P., Lensing A. W., Prins M. H., et al. (2004), "Below-knee elastic compression stockings to prevent the postthrombotic syndrome: a randomized, controlled trial", Ann Intern Med, 141 (4), pp. 249-56.

17. Rossi F. H., Volpato M. G., Metzger P. B., et al. (2015), "Relationships between severity of signs and symptoms and quality of life in patients with chronic venous disease", Jornal Vascular Brasileiro, 14, pp. 22-28.

18. Ruckley C. V. (1997), "Socioeconomic impact of chronic venous insufficiency and leg ulcers", Angiology, 48 (1), pp. 67-9.

19. Soydan E., Yilmaz E., Baydur H. (2016), "Effect of socio-demographic characteristics and clinical findings on the quality of life of patients with chronic venous insufficiency", Vascular, 25 (4), pp. 382-389.

20. Valencia I. C., Falabella A., Kirsner R. S., et al. (2001), "Chronic venous insufficiency and venous leg ulceration", J Am Acad Dermatol, 44 (3), pp. 401-21; quiz 422-4.

21. Vayssairat M., Ziani E., Houot B. (2000), "Placebo controlled efficacy of class 1 elastic stockings in chronic venous insufficiency of the lower limbs", J Mal Vasc, 25 (4), pp. 256-62. 